\title{
Infra-Red Plasmonic Sensors
}

\author{
Anthony Centeno ${ }^{1,3, *}$, Siti Rahmah Aid ${ }^{2}$ and Fang Xie ${ }^{3}$ \\ 1 Department of Electrical and Electronic Engineering, Xi'an Jiaotong Liverpool University, 111 Ren'ai Road, \\ Suzhou Dushu Lake Higher Education Town, Suzhou 215123, China \\ 2 Department of Electronic Systems Engineering, Malaysia Japan International Institute of Technology, \\ University Technology Malaysia, Kuala Lumpur 54100, Malaysia; sitirahmah.aid@utm.my \\ 3 Department of Materials, Imperial College London, Exhibition Road, London SW7 2AZ, UK; \\ f.xie@imperial.ac.uk \\ * Correspondence: Anthony.Centeno@xjtlu.edu.cn; Tel.: +86-512-8188-4766
}

Received: 30 November 2017; Accepted: 10 January 2018; Published: 16 January 2018

\begin{abstract}
Plasmonic sensors exploiting the localized surface plasmon resonance (LSPR) of noble metal nanoparticles are common in the visual spectrum. However, bio-sensors near the infra-red (NIR) windows (600-900 nm and 1000-1400 nm) are of interest, as in these regions the absorption coefficients of water, melanin deoxyglobin, and hemoglobin are all low. The first part of this paper reviews the work that has been undertaken using gold $(\mathrm{Au})$ and silver $(\mathrm{Ag})$ particles in metal enhanced fluorescence (MEF) in the NIR. Despite this success, there are limitations, as there is only a narrow band in the visual and NIR where losses are low for traditional plasmonic materials. Further, noble metals are not compatible with standard silicon manufacturing processes, making it challenging to produce on-chip integrated plasmonic sensors with Au or Ag. Therefore, it is desirable to use different materials for plasmonic chemical and biological sensing, that are foundry-compatible with silicon $(\mathrm{Si})$ and germanium $(\mathrm{Ge})$. One material that has received significant attention is highly-doped $\mathrm{Ge}$, which starts to exhibit metallic properties at a wavelength as short as $6 \mu \mathrm{m}$. This is discussed in the second part of the paper and the results of recent analysis are included.
\end{abstract}

Keywords: NIR; plasmonics; LSPR; MIR; germanium

\section{Introduction}

At the interface between materials with different signs for the real part of the permittivity, surface plasmon polaritons (SPPs) can be excited. The requirements for the negative permittivity material are normally met by noble metals, gold (Au) and silver $(\mathrm{Ag})$, since the wave is on the boundary of the metal and an external medium it is very sensitive to any change of this boundary. The excitation of surface plasmons by light is termed surface plasmon resonance. The resultant resonant interaction between the SPPs and the metal surface results in a significantly enhanced electromagnetic near-field [1]. SPPs have many applications in subwavelength optics, including in chemical sensors and biosensors [2,3].

If $\mathrm{Au}$ or Ag nanoparticles (of dimensions much smaller than the wavelength of excitation) are considered, incident light can excite localised surface plasmons (LSPs), where the charge density oscillations are confined to the metallic particles. An external field is able to displace the free electrons in the nanoparticle with respect to the fixed ionic core [1]. This displacement sets up a restoring force leading to coherent oscillations of the charge density, and hence, a resonant frequency. This is termed localised surface plasmon resonance (LSPR) and has been widely researched and applied in sensors [4-7], solar cells [8,9], and spectroscopy [10].

One application that exploits the LSPR, which has received much attention for the purpose of bio-sensing, is metal enhanced fluorescence (MEF) [11-28]. MEF is now a well-recognized form of technology wherein the near-field interaction of fluorophores with metallic nanostructures can lead to substantial fluorescence enhancement. 
Fluorescent molecules emitting at wavelengths in the infra-red window, in which penetration depth is high and the autofluorescence minimum is of particular interest, are potentially an attractive technology for bio-applications [27]. However, the low quantum yield and poor photostability of near infra-red (NIR) dyes currently limits their applicability. Design and synthesis of NIR dyes with high quantum yield and photostability have proved to be extremely challenging, due to the complex synthetic routes required for these large, complex molecules [27]. The amplification of light from NIR fluorophores by MEF is a promising strategy for dramatically improving both the detection sensitivity and image enhancement, thereby realizing the potential advantages of the NIR fluorophores. Section 2 of this paper discusses the physical process of MEF and reviews some of the published work by the authors.

NIR losses arise in Au and Ag from intraband (or Drude) losses. There is, therefore, only a narrow band in the visual and NIR range where losses are low for traditional plasmonic materials. A further challenge associated with noble metals is that they are not compatible with standard silicon manufacturing processes. Further, noble metals diffuse into the semiconductor, forming deep-level traps which have an adverse effect on device performance. While $\mathrm{Au}$ and $\mathrm{Ag}$ are the obvious choice for visible and NIR applications, there is a desire and need for chemical and biological sensing in the mid-infrared (MIR) range [29-31] using materials that are foundry-compatible with silicon (Si) and germanium (Ge), that might lead to on-chip integration of devices governed by plasmonic effects $[25,26]$. One material that has received significant attention as a potential plasmonic material in the MIR is highly-doped Ge $[29,32,33]$. In Section 3 of this paper we explore the advantages of highly-doped Ge as a MIR plasmonic material. By analyzing data available in the literature for doped Ge thin-films we discuss, using computational electromagnetics, some of the fundamental issues related to future applications and exploitation.

\section{Metal Enhanced Fluorescence in the Near Infra-Red}

MEF can be considered a three-stage process [24]. The first is the increased absorption of exciting light by the dye molecule due to the enhanced electric field around the nanoparticle, caused by the LSPR. Once in an excited state the molecule undergoes internal processes to bring it into the emitted excited state. Although the metal can modify these processes, they are very fast compared to the other two processes, and are not usually considered in the analysis of the MEF mechanism. Finally the molecule decays, through the emission of a photon, to the ground state. The metal will modify the radiative decay rate and create new channels of non-radiative decay, through energy and charge transfer between the molecule and metal.

The fluorescence rate, $\Psi$, is the product of the excitation rate, $\gamma_{e}$, and the quantum yield, $\varphi$. At the excitation wavelength of the molecule, $\lambda_{\mathrm{ex}}$, the incident light irradiates the metal nanoparticle and the near-field around the particle excites the emitter. At the emission wavelength, $\lambda_{\mathrm{em}}$, of the fluorophore it behaves as an oscillating dipole. Since it is in the proximity of the metal nanoparticle the radiated emissions from the fluorophore, and hence the quantum yield, are modified [34].

The quantum yield of an isolated fluorophore molecule is [34]:

$$
\phi^{0}=\frac{\gamma_{r}^{0}}{\gamma_{r}^{0}+\gamma_{n r}^{0}}
$$

where $\gamma_{r}^{0}$ and $\gamma_{n r}^{0}$ are the radiative and non-radiative decay rates respectively. The superscript 0 indicates the fluorophore is isolated, rather than in the presence of a metal nanoparticle.

In the presence of a metallic particle there will be additional radiative and absorption channels, giving a modified quantum yield [35]:

$$
\phi^{m}=\frac{\gamma_{r}^{m}}{\gamma_{r}^{m}+\gamma_{a b s}^{m}+\gamma_{n r}^{0}}
$$


We consider a single fluorophore that is coupled to a nanoparticle to obtain values of modified quantum yield and fluorescent rate enhancement. This requires a calculation of the decay rates $\gamma_{r}^{m}$, $\gamma_{a b s}^{m}$, and $\gamma_{r}^{0}$ by considering the spontaneous emission of the fluorophore as a small electric dipole [35]. These decay rates can be found in terms of the Poynting vector, as described in [35], such that:

$$
\gamma_{\mathrm{r}}=\frac{\int_{\mathrm{s}} \operatorname{Re}\left(\mathbf{E}_{\mathbf{T}} \times \mathbf{H}_{\mathbf{T}}{ }^{*}\right) \mathrm{da}}{2}
$$

and

$$
\gamma_{a b s}^{m}=\frac{-\int_{\mathrm{s}} \operatorname{Re}\left(\mathbf{E}_{\mathbf{S}} \times \mathbf{H}_{\mathbf{S}}{ }^{*}\right) \mathrm{da}}{2}
$$

where $\mathrm{s}$ is a surface that encloses the fluorophore molecule (small dipole) and nanoparticle. In Equation (3) we consider the total electric $\left(\mathbf{E}_{\mathbf{T}}\right)$ and magnetic field $\left(\mathbf{H}_{\mathbf{T}}\right)$ crossing $\mathbf{s}$, whereas in Equation (4) it is the scattered fields $\left(\mathbf{E}_{\mathbf{S}}\right.$ and $\mathbf{H}_{\mathbf{S}}$ ) from the nano-cylinder that are considered, and hence the subscripts T and S. To find $\gamma_{r}^{0}$ from Equation (3) only the small dipole has to be considered in the calculation, while to find $\gamma_{r}^{m}$ the metal nano-cylinder is added to the model and enclosed by the surface.

The excitation rate is found by considering the local electric field at the position and wavelength of excitation, $\mathbf{E}\left(\mathrm{x}_{\mathrm{d}}, \lambda_{\mathrm{ex}}\right)$ and the emitters orientation, $\mathbf{e}_{\mathbf{p}}$. If we consider the electric field in the presence of the metal nanoparticle near the fluorophore molecule, then the excitation rate enhancement is [36]:

$$
\chi=\frac{\gamma_{e}^{m}}{\gamma_{e}^{0}}=\frac{\left|\mathrm{E}\left(\mathrm{x}_{\mathrm{d}}, \lambda_{\mathrm{ex}}\right) \cdot \mathrm{e}_{\mathrm{p}}\right|^{2}}{\left|\mathrm{E}_{\mathrm{i}}\right|^{2}}
$$

where $\mathbf{E}_{\mathbf{i}}$ is the free space electric field (incident field) without the nano-cylinder being present.

The fluorescent rate enhancement, $\Psi_{e n h}$, can now be found from:

$$
\Psi_{\text {enh }}=\chi \cdot \frac{\varphi^{m}}{\varphi^{0}}
$$

From Equations (1)-(6) it is possible to calculate the emission enhancement from a fluorophore in close proximity to metal particle, using computational electromagnetics $[1,35,36]$.

Figure 1 shows the calculated electric field enhancement around cylindrical nanoparticles for an incident plane wave of wavelength $650 \mathrm{~nm}$. It can be seen that in most of the gap the magnitude of the electric field exceeds that of the incident field (the scale is logarithmic). This leads to excitation enhancement of fluorophores in this region. In the proximity of the corner, at the top of the nano-cylinder, the magnitude of the near-field is seen to exceed that of the incident field by two orders of magnitude. Previously published work used finite difference time domain (FDTD) analysis to calculate both the excitation and emission enhancement of fluorophores. The results showed that it is important, for the maximum fluorescent rate enhancement that the excitation and wavelengths of the dye should be above the absorption maxima for the nanoparticle array [1,35].

The NIR windows (600-900 $\mathrm{nm}$ and 1000-1400 nm) are of interest as they are regions where the absorption coefficients of water, melanin deoxyglobin, and hemoglobin are all low. Most of the reported works on MEF for the enhancement of NIR fluorescent dyes are based on gold nanostructures including nanorods, nanoshells and porous Au films by dealloying [27]. This is primarily because Au has a lower plasma frequency than Ag, so the LSPR peak is at a longer wavelength, as well as having a higher chemical stability. However, Ag can be an attractive material since it has a lower absorption efficiency and a higher scattering efficiency. This leads to larger field enhancement at the LSPR, leading to a larger excitation enhancement in MEF. The LSPR is dependent not just upon material but also on the shape of the nanoparticle, and MEF has been successfully demonstrated in the NIR using triangular-like Ag nanoparticles immobilized on glass substrate [26,27], nanocylinders [22], and Nanostar [23]. Table 1 
summarizes some of the results presented in published work by the Xie group at Imperial College for MEF in the NIR, for both $\mathrm{Au}$ and Ag nanoparticles, for excitation wavelengths up to $780 \mathrm{~nm}$. It should be noted that the emission rate enhancement is limited by the unmodified quantum yield. If the fluorescent molecule already has a high unmodified quantum yield, then the emission rate enhancement will be low. NIR fluorophores have relatively low unmodified quantum yields, typically less than 0.1 . Hence, emission rate enhancements of an order of magnitude are obtainable, although excitation rate enhancement is the main contributor to overall fluorescent enhancement.
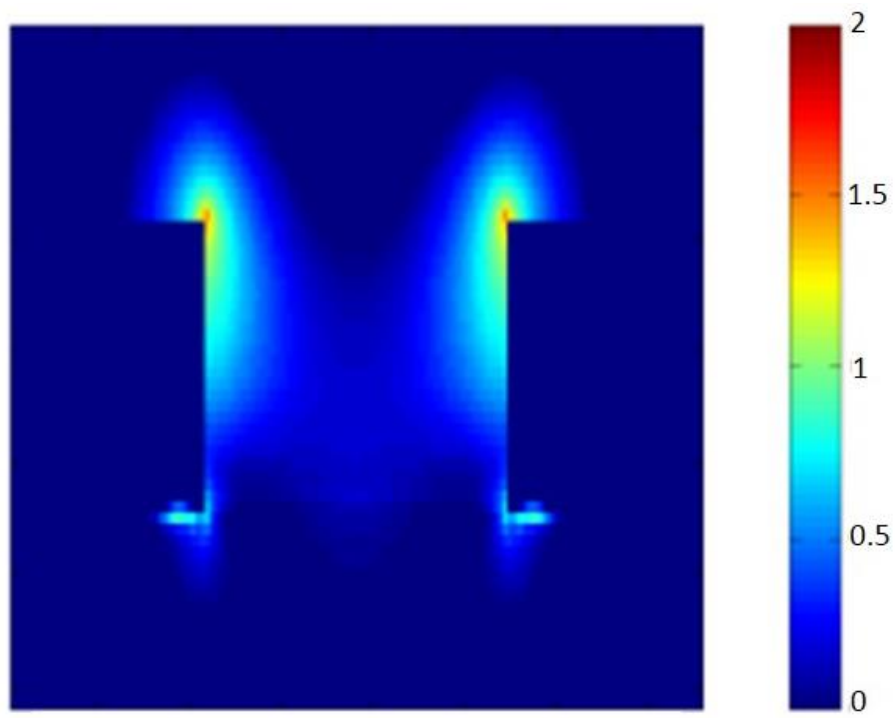

Figure 1. Electric field in the gap between two gold cylindrical nanoparticles for an incident plane wave of wavelength $650 \mathrm{~nm}$. The nanoparticles are in a two-dimensional hexagonal array and have a diameter of $170 \mathrm{~nm}$, height $50 \mathrm{~nm}$, and center-to-center separation of $300 \mathrm{~nm}$. The electric field magnitude is normalized to the magnitude of the incident field. Note the scale is logarithmic from 0 (dark blue) to 2 (dark red).

Table 1. Metal enhanced fluorescence in the near infra-red using gold ( $\mathrm{Au}$ ) and silver (Ag) nanoparticles. The excitation and emission rate enhancements are found from lifetime measurements and are not available in every case.

\begin{tabular}{|c|c|c|c|c|c|c|c|}
\hline Fluorophore & $\begin{array}{c}\text { Excitation } \\
\text { Wavelength } \\
(\mathrm{nm})\end{array}$ & $\begin{array}{c}\text { Emission } \\
\text { Wavelength } \\
(\mathrm{nm})\end{array}$ & $\begin{array}{c}\text { Fluorescent } \\
\text { Enhancement Factor }\end{array}$ & $\begin{array}{c}\text { Excitation Rate } \\
\text { Enhancement } \\
(\chi)\end{array}$ & $\begin{array}{c}\text { Emission Rate } \\
\text { Enhancement } \\
\qquad\left(\varphi^{m} / \varphi^{\circ}\right)\end{array}$ & $\begin{array}{c}\text { Type of } \\
\text { Nanoparticle }\end{array}$ & Reference \\
\hline AF 790 & 780 & 790 & 68.8 & - & - & $\mathrm{Au}$, Nanotriangle & [26] \\
\hline AF 750 & 730 & 750 & 235 & 25.2 & 9.3 & $\mathrm{Au}, \mathrm{Cylinder}$ & [22] \\
\hline AF 750 & 730 & 750 & 321 & 107 & 3 & $\mathrm{Au}$, Nanostar & [23] \\
\hline $\mathrm{Ag}_{2} \mathrm{QD}$ & 780 & 1205 & 40 & - & - & $\mathrm{Au}$, Nanostar & [23] \\
\hline
\end{tabular}

The geometry and dimensions of the nanoparticles presented in Table 1 are summarized. The Au Nanostar had a core size of $134 \mathrm{~nm}$, with spike length $60 \mathrm{~nm}$. The Au cylinders had a diameter of $256 \mathrm{~nm}$ and height $50 \mathrm{~nm}$ and were immobilized on a glass slide, in a hexagonal array with center-to-center separation of $300 \mathrm{~nm}$. The nanotriangles were also immobilized in hexagonal arrays in glass slides. The Ag ones had sides of $208 \mathrm{~nm}$ and tip-to-tip separation of $60 \mathrm{~nm}$. The Au ones had sides of $141 \mathrm{~nm}$ and tip-to-tip separation of $17.7 \mathrm{~nm}$. The main contribution to the fluorescent enhancement is the increased electric field, due to the LSPR extinction peak, causing excitation rate enhancement. The position of the LSPR extinction peak is a due to a number of factors, including nanoparticle material, particle shape and size, inter-particle separation and the surrounding dielectric 
medium. Computational electromagnetic modelling is carried out to find the extinction peaks and field enhancement due to the metal nanoparticles. For a full description of the particle geometries readers should refer to the references provided in Table 2.

\section{Ge as a Plasmonic Material in the Mid Infra-Red}

Germanium $(\mathrm{Ge})$ is a promising material for replacing silicon as a substrate for metal oxide semiconductor (MOS) devices. Further downscaling of silicon-based devices will lead to the short channel effect (SCE) that will result in an increase in the leakage current [37-39]. This leakage current will increase the power consumption of devices, while also reducing the performance. High drive current capability of devices without further downscaling process can be realized by increasing the carrier mobility in the substrate. Ge has emerged as one of the potential candidates to replace Si as a substrate for MOS transistors, due to its higher electrical carrier mobility $\left(3900 \mathrm{~cm}^{2} / \mathrm{V} \cdot \mathrm{s}\right.$ for electrons and $1900 \mathrm{~cm}^{2} / \mathrm{V} \cdot \mathrm{s}$ for holes) [37]. Furthermore, its similarity with conventional Si will ease the replacement process in manufacturing lines. It would therefore be very attractive if plasmonic sensors could be based on Ge for on-chip integration.

To consider this further we start from the free electron response in metals, given by the well-known Drude model:

$$
\varepsilon(\omega)=\varepsilon^{\prime}+\varepsilon^{\prime \prime}=\varepsilon_{\infty}-\frac{\omega_{p}^{2}}{\omega^{2}+i \omega \gamma}=\varepsilon_{\infty}-\frac{\omega_{p}^{2}}{\omega^{2}+\gamma^{2}}+i \frac{\omega_{p}^{2} \gamma}{\left(\omega^{2}+\gamma^{2}\right) \omega}
$$

where $\varepsilon_{\infty}$ is the high frequency relative permittivity, $\omega_{p}$ is the plasma frequency, and $\gamma$ is the Drude relaxation rate.

The optical response of free carriers is described by Equation (7). If we consider the real part and define a cross-over frequency, $\omega_{c}$, where the real part becomes zero, we get:

$$
\omega_{p}^{2}=\varepsilon_{\infty}\left(\omega_{C}^{2}+\gamma^{2}\right)
$$

Rearranging (8) gives:

$$
\omega_{c}=\sqrt{\frac{\omega_{p}^{2}}{\varepsilon_{\infty}}-\gamma^{2}}
$$

This is the shortest wavelength where the semiconductor can exhibit metal like properties, that is have a negative real part of permittivity. The required free carrier concentration, $n$, in the semiconductor for a cross-over frequency can be found from [33]:

$$
n=\frac{\omega_{p}^{2} m^{*}}{4 \pi \varepsilon_{0} e^{2}}
$$

where $\varepsilon_{0}$ is the free space permittivity, $m^{*}$ is the effective mass of the carrier and $e$ is the electron charge.

As can be seen the higher the free carrier concentration the higher the cross-over frequency. It should be noted here that in the literature it is often assumed that the plasma frequency and the cross-over frequency are the same. In fact this is only the case for the lossless case where $\gamma=0$ and if interband transitions are ignored $\left(\varepsilon_{\infty}=1\right)$. This is discussed in depth by Frigerio et al. [33]. To fit the Drude model to the dielectric function the value of $\varepsilon_{\infty}$ is approximately the dielectric constant of undoped semiconductor in the $\operatorname{MIR}$ ( $\approx 16$ for $\mathrm{Ge}$ ).

In the visual-NIR the analysis and design of plasmonic devices requires accurate determination of the dielectric function. This is then used to find the electromagnetic field, or find the dispersion relation, using computational electromagnetics. To do this the parameters $\varepsilon_{\infty}, \omega_{p}$, and $\gamma$ need to be found and applied in an electromagnetic simulation. The parameters can be found by using a multilayer-based model to calculate the reflectance from the doped Ge film and iteratively modifying the parameters to obtain a good fit to measurements. These can be obtained in the MIR using Fourier transform 
infra-red (FTIR) spectroscopy (rather than UV-VIS-NIR spectroscopy). The calculation of reflection can be done using the transfer matrix method [40] [see for example the code from Steven Byrnes at http://sjbyrnes.com]. However, since the reflectivity measurements are made on a thin film it is very fast to calculate reflection using a finite difference time domain (FDTD) model, as the only spatial discretization required is in the direction of the incident plane wave, which is normal in this case. The advantage of using FDTD is that the Drude model can be implemented directly [41].

In this work, we have first considered published data from Frigerio et al. [33] and Prucnel et al. [29]. In both cases we derive the Drude model from their experimental data. We also fit data from FTIR measurements we have undertaken on Ge thin films manufactured using ion implantation and rapid laser annealing [37].

Frigerio et al. [33] have considered heavily-doped films produced using a low-energy-plasma-enhanced chemical vapor deposition (CVD) reactor, using phosphorus as the n-type dopant. They present the dielectric functions for samples with carrier densities $(\eta)$ up to $3.0 \times 10^{19} \mathrm{~cm}^{-3}$. We have then used the RefFIT code [42] to extract the parameters for the Drude model for two samples, shown in Table 2. These are then used in an FDTD code [43] to calculate the extinction properties and electric field enhancements.

Table 2. Drude parameters for phosphorus-doped Ge derived, from [33] using RefFIT [42]. The thicknesses of the germanium films reported in [33] are of $2 \mu \mathrm{m}$.

\begin{tabular}{ccccccc}
\hline Material Number & $\eta\left(\mathbf{c m}^{-\mathbf{3}}\right)$ & $\varepsilon_{\infty}$ & $\omega_{p}\left(\mathbf{c m}^{-\mathbf{1}}\right)$ & $\omega_{\mathcal{c}}\left(\mathbf{c m}^{-\mathbf{1}}\right)$ & $\gamma\left(\mathbf{c m}^{-\mathbf{1}}\right)$ & Sample Number in Reference [33] \\
\hline 1 & $2.3 \times 10^{19}$ & 16.5 & 4032 & 974.3 & 189.83 & 9338 \\
2 & $3.0 \times 10^{19}$ & 16.2 & 4705 & 1147.4 & 224.64 & 9336 \\
\hline
\end{tabular}

Using these parameters we have investigated the spectral response of a rectangular prism-doped germanium ( $\mathrm{n}-\mathrm{Ge}$ ) particles sitting on an un-doped Ge substrate, as depicted in Figure 2. Figure 3 shows the absorption, scattering and extinction for a particle that is $2-\mu \mathrm{m}$ long, $1-\mu \mathrm{m}$ wide and $1-\mu \mathrm{m}$ high. It can be seen that there are two extinction peaks, one at $490 \mathrm{~cm}^{-1}$ (wavelength of $20.4 \mu \mathrm{m}$ ) and the other at $1050 \mathrm{~cm}^{-1}(9.523 \mu \mathrm{m})$.

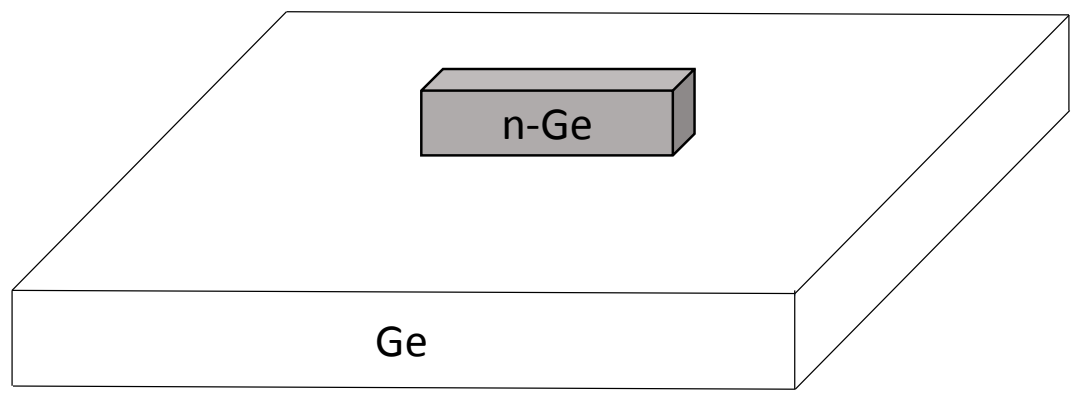

Figure 2. Depiction of the model used for the finite difference time domain (FDTD) calculations. Here, a phosphorous doped, n-type germanium (n-Ge) rectangular particle on top of a germanium (Ge) substrate is shown.

Considering the extinction peaks in Figure 3 it can be seen that the lower frequency peak, although predominantly absorption, also has a significant scattering content. On the other hand, the higher frequency peak is absorption dominated. Figure 4 shows the electric field enhancement at both frequencies. It can be seen from Figure $4 \mathrm{a}$ that at $490 \mathrm{~cm}^{-1}$ the peak field enhancement is of two orders of magnitude at the corners of the n-Ge particle, at the interface with the Ge substrate. Nevertheless at least an order of magnitude $(\times 10)$ enhancement is seen around the sides of the particle. In contrast the field enhancement is much lower at $1049 \mathrm{~cm}^{-1}$, with a maximum of 1 order of magnitude very close to the n-Ge surface. The sensitivity of an LSPR sensor is related to the increased electric field enhancement around the particle. 


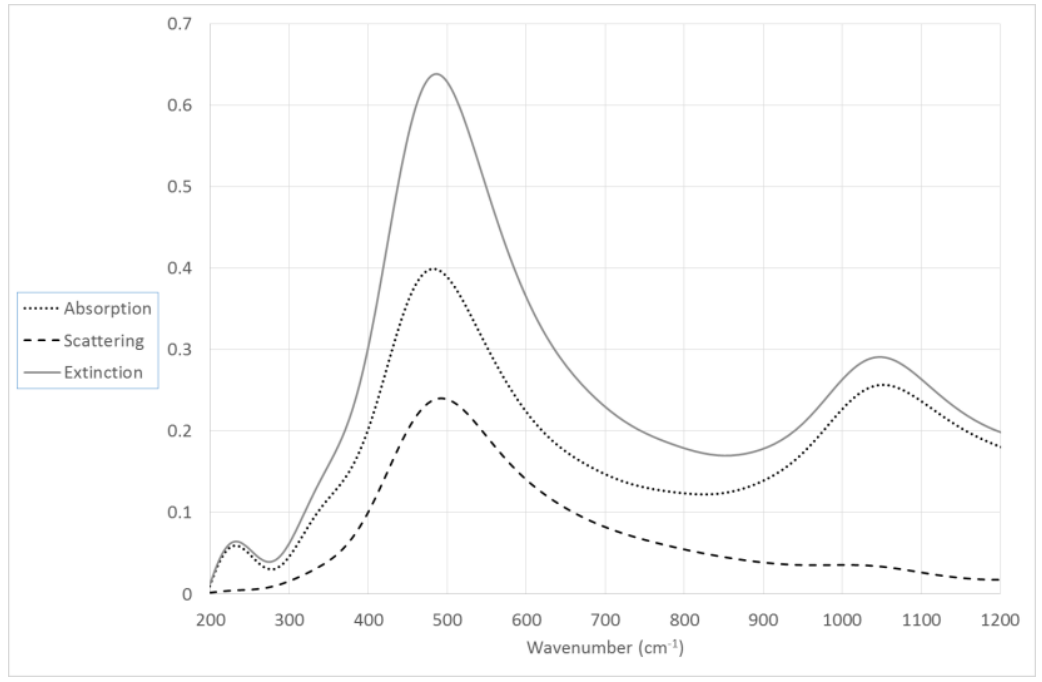

Figure 3. Spectral response for rectangular prism shaped n-type doped germanium (n-Ge) particle (material 2 in Table 2) calculated using FDTD. Height $1 \mu \mathrm{m}$, length $2 \mu \mathrm{m}$, width $1 \mu \mathrm{m}$.

The LSPR mode at $490 \mathrm{~cm}^{-1}$ is strongly influenced by the undoped Ge substrate. This causes strong field enhancement at the lower corners of the n-Ge particle, where it has an interface with both air and Ge. There is also strong field enhancement within the Ge substrate. The high refractive index of the Ge also causes this mode to have a low frequency.

At the higher wavenumber the resonance is close to the cut-off wavelength $\left(\omega_{c}\right)$ that can support surface plasmon Polaritons and hence an LSPR. This means that the magnitude of the real part of the permittivity is small at this wavelength. There is negligible field enhancement in the Ge substrate causing this mode to have a higher frequency.

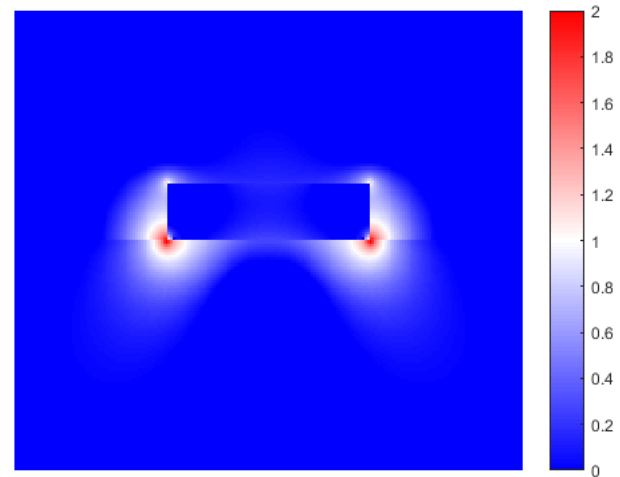

(a)

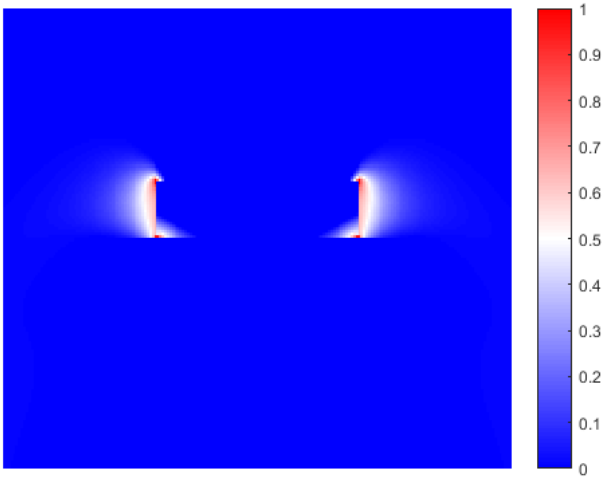

(b)

Figure 4. Electric field enhancement the extinction peaks of a 1- $\mu \mathrm{m}$-high highly doped germanium (n-Ge) particle. The excitation is (a) $490 \mathrm{~cm}^{-1}$ and (b) $1049 \mathrm{~cm}^{-1}$, respectively. The scale is logarithmic and the electric field enhancement is normalized to the incident electric field. The plots are for a cross-section through the center of the particle (mid-width) (The incident field is normal to the top surface of the particle and polarized in the length direction).

The height of the n-Ge particle considered in the model was reduced to $0.5 \mu \mathrm{m}$ and $0.1 \mu \mathrm{m}$, respectively. The spectral response is shown in Figure 5 for both FDTD calculations. It can be seen that the longer wavelength extinction peak is less much less pronounced for the $0.5-\mu \mathrm{m}-\mathrm{high} n-\mathrm{Ge}$ particle, while for the $0.1-\mu \mathrm{m}$ case there is only a single observable peak, which is caused almost totally by absorption. 


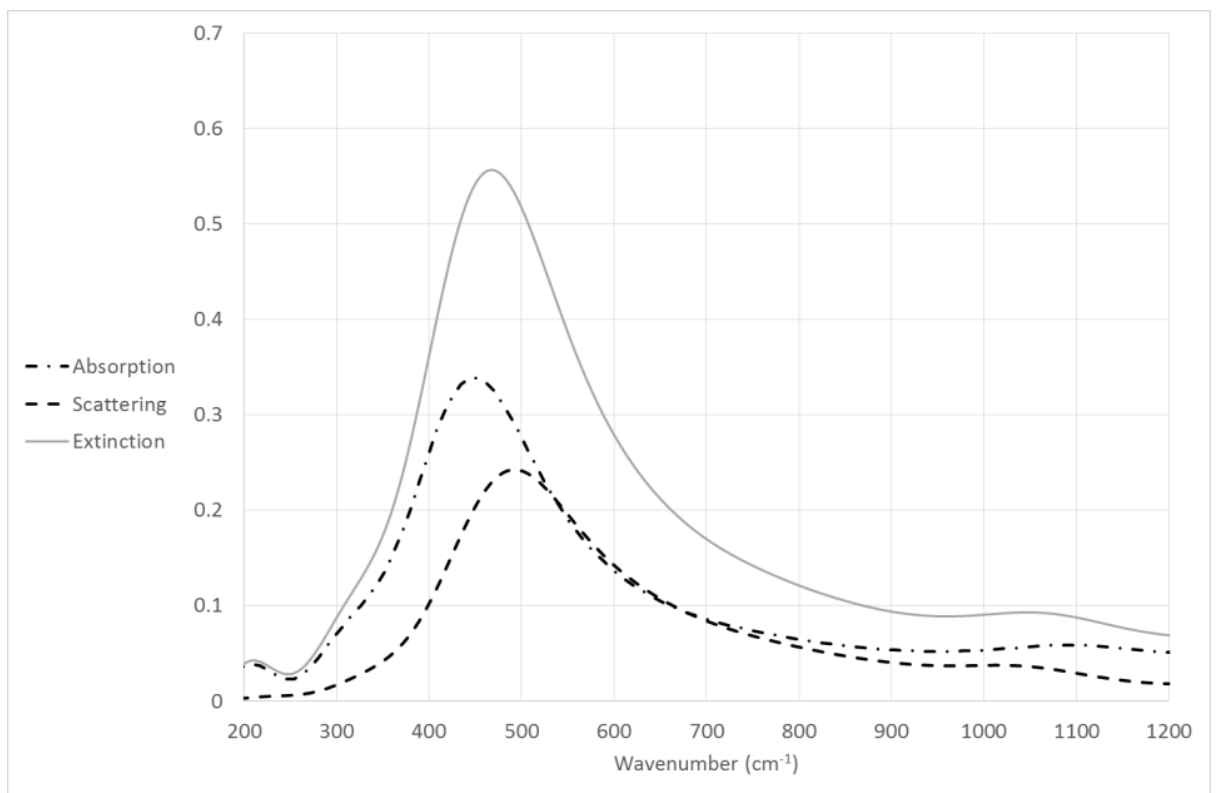

(a)

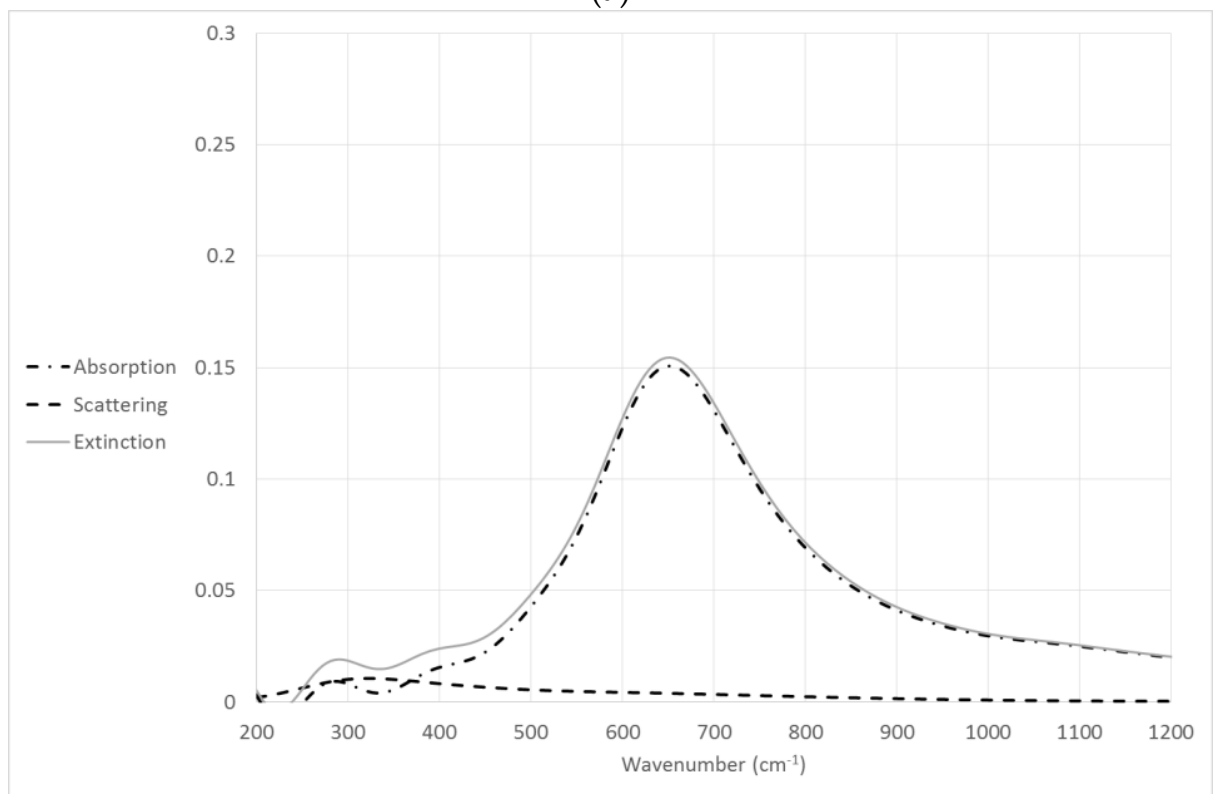

(b)

Figure 5. Spectral response for rectangular doped Ge particles (material 2 in Table 2) for (a) height $0.5 \mu \mathrm{m}$ and (b) height $0.1 \mu \mathrm{m}$. The length is $2 \mu \mathrm{m}$ and the width is $1 \mu \mathrm{m}$ in both cases.

The electric field enhancement for the $0.1 \mu \mathrm{m}$ high n-Ge particle at $651 \mathrm{~cm}^{-1}$ is shown in Figure 6. The enhancement is much less than 1 order of magnitude and strongly confined to the sides of the particle. This suggests that the use of $n-G e$ as LSPR sensors is limited by their height.

A higher carrier (doping) density is obtainable using ion implantation and annealing. Prucnal et al. [22] achieved carrier concentrations of $\sim 2.2 \times 10^{20}$ using rear side flash-lamp annealing (r-FLA). This gave n-Ge films of thickness $140 \mathrm{~nm}$. Our analysis, based on their reflection measurements, gave a $\omega_{c}$ of $1850 \mathrm{~cm}^{-1}$ (wavelength $=5.4 \mu \mathrm{m}$ ). Our group has produced $\mathrm{n}$-Ge films from ion implantation and rapid laser thermal annealing [37]. The thickness of these films is between 40 and $90 \mathrm{~nm}$ on a Ge substrate. FDTD models were used to replicate the reflection obtained from FTIR measurements. From this analysis it was found that an $\mathrm{n}-\mathrm{Ge}$ film had the following Drude parameters: $\omega_{p}=6500 \mathrm{~cm}^{-1}, \varepsilon_{\infty}=16.5, \gamma=241 \mathrm{~cm}^{-1}$. The comparison between the experimental and 
fitted results is shown in Figure 7. The analysis gives a $\omega_{c}$ of $1582 \mathrm{~cm}^{-1}$ (wavelength $6.3 \mu \mathrm{m}$ ) and a carrier concentration of $5.5 \times 10^{19} \mathrm{~cm}^{3}$. (Reference [37] should be referred to for further discussion of the fabrication process).

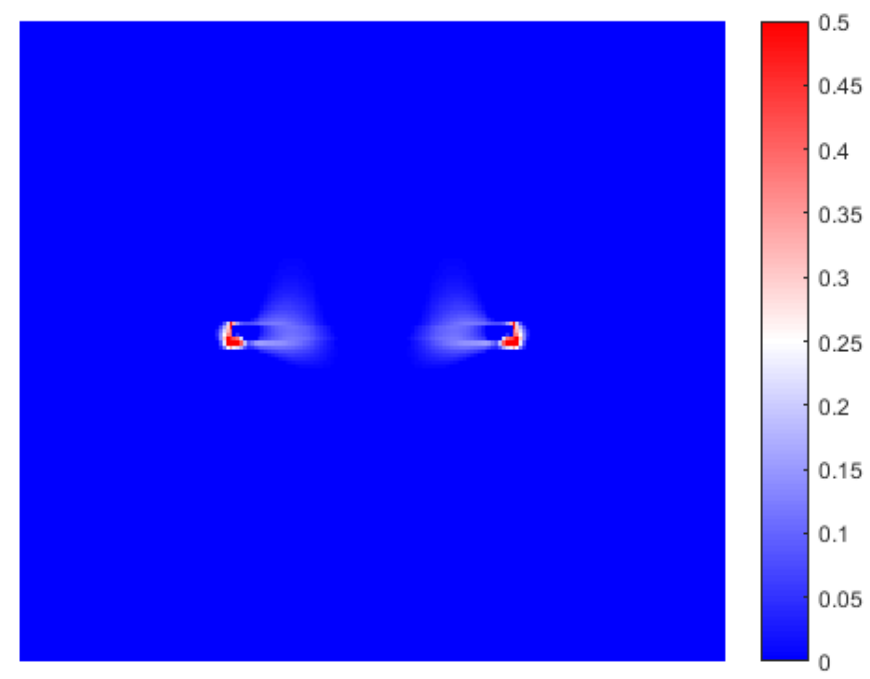

Figure 6. Electric field enhancement for extinction peak of $0.1 \mu \mathrm{m}$ high $\mathrm{n}$-Ge particle. The scale is logarithmic and the electric field enhancement is normalized to the incident electric field.

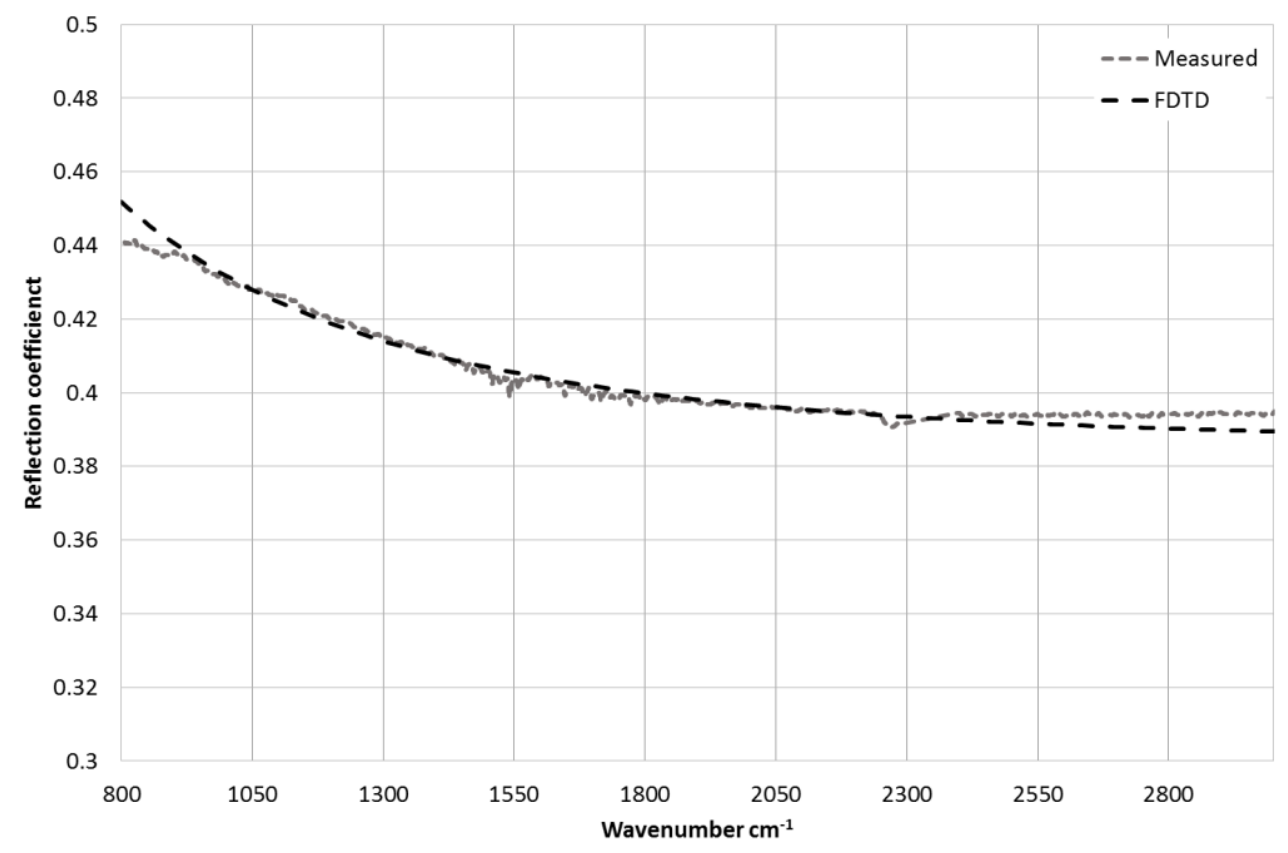

Figure 7. Measured and modelled reflection from a phosphorous doped germanium (n-Ge) thin film of thickness $40 \mathrm{~nm}$, and cut-of frequency $\left(\omega_{\mathcal{c}}\right) 1582 \mathrm{~cm}^{-1}$.

These results show that n-Ge films can be produced with large carrier concentrations and cross-over wavelengths around $6 \mu \mathrm{m}$. Unfortunately, the thickness of the films is only of the order of $100 \mathrm{~nm}$, or less, too thin to support large field enhancement from the LSPR of a particle. Nevertheless, it may be possible that the thin film can be used to support propagating surface plasmon polaritons for a plasmonic MIR sensor. The production of $n-G e$ films, with a high $\omega_{c}$, is an important first step along this path but significant research is needed in the future to produce integrated plasmonic sensors. 
The real and imaginary parts of the dielectric constant derived from the two materials considered in Table 2 are shown in Figure 8, where the frequency ( $x$-axis) is normalized to $\omega_{p}$ for both samples. It is interesting to note that the real and imaginary parts for both materials overlay each other when normalized. Hence, the results shown in Figures 3-6 could also be normalized (or scaled in dimensions) to the plasma frequency, which is proportional to the free carrier concentration.

We carried out a similar exercise for $\mathrm{n}-\mathrm{Ge}$ samples produced by ion-implantation and rapid laser thermal annealing, rather than CVD, where the annealing conditions were different in each case. It was found that in these cases there was significant variation in the normalized values of permittivity between different samples. This was expected as the ion implantation induces considerable damage to the crystalline germanium, producing an amorphous layer at the surface. The recrystallization of the doped germanium, to produce crystalline n-Ge with high carrier activation, is strongly dependent on the annealing conditions [37]. Annealing conditions also affects the diffusion of n-type dopant into the Ge substrate material.

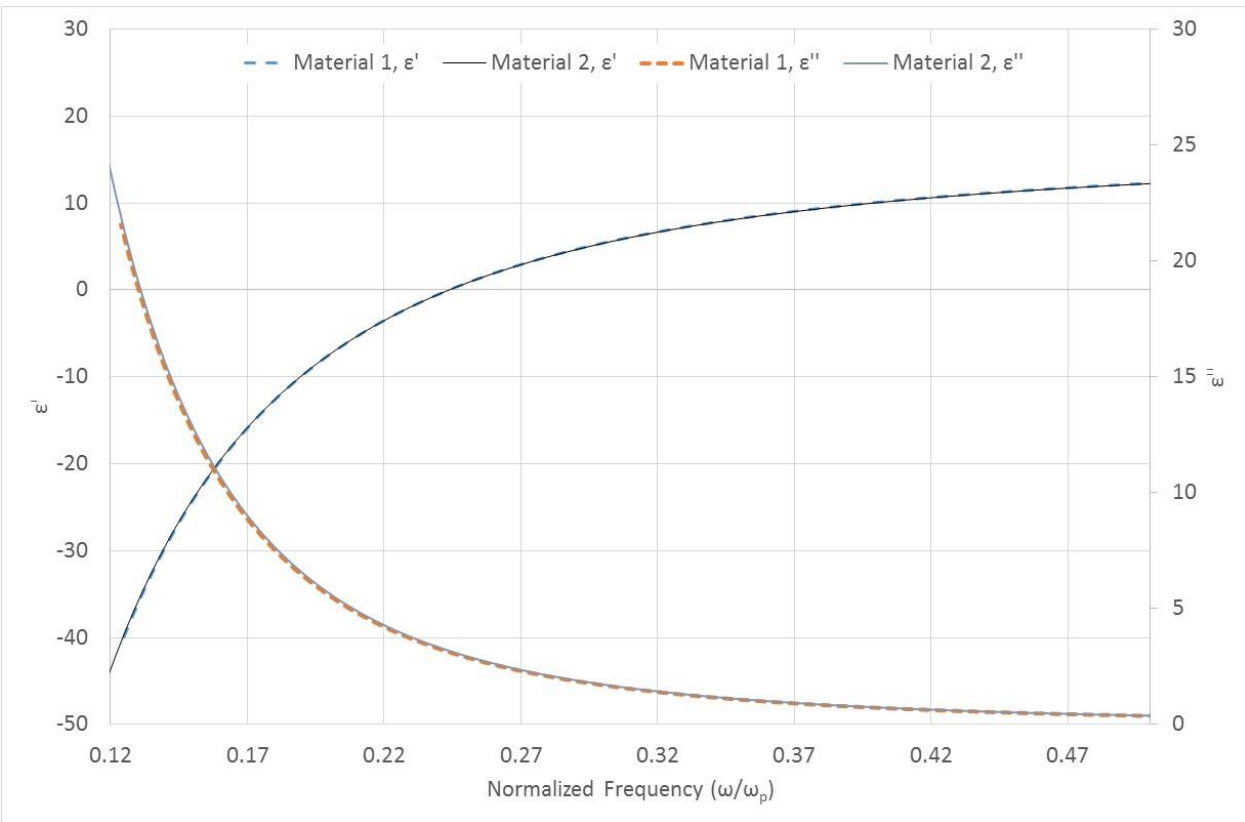

Figure 8. Real and Imaginary parts of the dielectric constant for materials number 1 and 2 in Table 2 (Samples 9338 and 9336 in reference [33]). The frequency is normalized to the plasma frequency. For these chemical vapor deposition (CVD) fabricated samples it is seen that the normalized permittivities overlay each other, enabling scaling.

\section{Discussion}

In this paper we have discussed the application of the localized surface plasmon resonance to infra-red chemical and biological sensors. Using metal enhanced fluorescence (MEF) it has been demonstrated that traditional plasmonic materials, $\mathrm{Au}$ and $\mathrm{Ag}$, can be used in the NIR window. The excitation wavelength of the LSPR, though, is limited to short wavelengths in the NIR ( 780 nm) as intraband (or Drude) losses become increasingly large. In addition, it would be very attractive to have on-chip integration of plasmonic sensors using materials that are compatible with silicon and germanium. Germanium is a promising material for replacing silicon as a substrate for MOS devices so the use of highly doped n-type germanium as a plasmonic material would be very beneficial.

While carrier densities in phosphorous doped germanium are high enough for cross-over frequencies with wavelengths shorter than $6 \mu \mathrm{m}$ the thickness of these films are only around $100 \mathrm{~nm}$ or less. This is too thin to enable the fabrication of LSPR sensors with large field enhancement. This is a fundamental limitation, because such high carrier densities are obtained using ion implantation and 
some form of rapid annealing. The thickness of the film can be increased, by increasing the annealing times, but this would result in a lower carrier density and a subsequent decrease in the cross-over frequency. Further electromagnetic modelling based on films presented in the literature produced using low-energy-plasma-enhanced chemical vapor deposition (CVD) indicate that low frequency LSPR modes have stronger field enhancement. This suggests that there are significant challenges associated in the development of LSPR sensors for wavelengths shorter than $20 \mu \mathrm{m}$. SPP could be supported at the shorter wavelengths, although this will present significant design challenges in exciting the SPP and integrating the sensors onto an on-chip platform.

\section{Conclusions}

$\mathrm{Au}$ and Ag have been successfully used for plasmonic sensors in the visual and NIR. MIR plasmonic sensors are much more challenging, but the initial work that has been carried out on highly doping Ge substrates. Calculations of the cross-over wavelength, indicate that fully integrated MIR plasmonic sensors are feasible for n-Ge material.

Acknowledgments: This work has been supported by an Imperial College Global Engagement Grant, Takasago Research Grant (4B211/4B273), Ministry of Education Malaysia, Universiti Teknologi Malaysia Research University Grant Tier 1 (14H01) and a British Council Newton Grant (\#216239013). We would like to acknowledge Hiroshi Ikenoue, Department of Gigaphoton Next GLP, Kyushu University, Japan for undertaking the rapid laser annealing process in the production of our n-Ge films.

Conflicts of Interest: The authors declare no conflict of interest.

\section{References}

1. Centeno, A.; Xie, F. An electromagnetic study of metal enhanced fluorescence due to immobilized particle arrays on glass substrate. Mater. Today Proc. 2015, 2, 94-100. [CrossRef]

2. Wijaya, E.; Lenaerts, C.; Maricot, S.; Hastanin, J.; Habraken, S.; Vilcot, J.-P.; Boukherroub, R.; Szunerits, S. Surface plasmon resonance-based biosensors: From the development of different SPR structures to novel surface functionalization strategies. Curr. Opin. Solid State Mater. Sci. 2011, 15, 208-224. [CrossRef]

3. Pranveer, S. SPR Biosensors: Historical Perspectives and Current Challenges. Sens. Actuators B Chem. 2016, 229, 110-130.

4. Ashaduzzaman, Md.; Deshpande, S.R.; Murugan, N.A.; Misra, Y.K.; Turner, A.P.F.; Tiwari, A. On/off-switchable LSPR nano-immunoassay for troponin-T. Sci. Rep. 2017, 7, 44027. [CrossRef] [PubMed]

5. Kumar, M.; Reddy, G.B. Stability-Inspired Entrapment of Ag Nanoparticles in $\mathrm{ZrO}_{2}$ Thin films. Plasmonics 2016, 11, 261. [CrossRef]

6. Kumar, M.; Jangid, T.; Panchal, V.; Kumar, P.; Pathak, A. Effect of Grazing Angle Cross-Ion Irradiation on Ag Thin Films. Nanoscale Res. Lett. 2016, 11, 454. [CrossRef] [PubMed]

7. Kumar, M.; Reddy, G.B. Tailoring surface plasmon resonance in $\mathrm{Ag}: \mathrm{ZrO}_{2}$ nanocomposite thin films. Phys. E 2010, 43, 470-474. [CrossRef]

8. Catchpole, K.R.; Polman, A. Plasmonic solar cells. Opt. Express 2008, 16, 21793-21800. [CrossRef] [PubMed]

9. Atwater, H.A.; Polman, A. Plasmonics for improved photovoltaic devices. Nat. Mater. 2010, 9, $205-213$. [CrossRef] [PubMed]

10. Willets, K.A.; Van Duyne, R.P. Localised surface plasmon resonance spectroscopy and sensing. Annu. Rev. Phys. Chem. 2007, 58, 267-297. [CrossRef] [PubMed]

11. Xie, F.; Baker, M.S.; Goldys, E.M. Homogeneous silver-coated nanoparticles for enhanced fluorescence detection. J. Phys. Chem. B 2006, 110, 23085-23091. [CrossRef] [PubMed]

12. Lakowicz, J.R. Radiative Decay Engineering: Biophysical and Biomedical Applications. Anal. Biochem. 2001, 291, 1-24. [CrossRef] [PubMed]

13. Lakowicz, J.R. Radiative decay engineering 5: Metal-enhanced fluorescence and plasmon emission. Anal. Biochem. 2005, 337, 171-194. [CrossRef] [PubMed]

14. Geddes, C.D.; Parfenov, A.; Roll, D.; Fang, J.; Lakowicz, J.R. Electrochemical and Laser Deposition of Silver for Use in Metal-Enhanced Fluorescence. Langmuir 2003, 19, 6236-6241. [CrossRef] [PubMed] 
15. Lakowicz, J.R.; Geddes, C.D.; Gryczynski, I.; Malicka, J.; Gryczynski, Z.; Aslan, K.; Lukomska, J.; Matveeva, E.; Zhang, J.; Badugu, R.; et al. Advances in Surface-Enhanced Fluorescence. J. Fluoresc. 2004, 14, 425-441. [CrossRef] [PubMed]

16. Pribik, R.; Dragan, A.I.; Zhang, Y.; Gaydos, C.; Geddes, C.D. Metal-Enhanced Fluorescence (MEF): Physical characterization of Silver-island films and exploring sample geometries. Chem. Phys. Lett. 2009, 478, 70-74. [CrossRef] [PubMed]

17. Yang, Z.; Ni, W.; Kou, K.; Zhang, S.; Sun, Z.; Sun, L.-D.; Wang, J.; Yan, C.-H. Incorporation of Gold Nanorods and Their Enhancement of Fluorescence in Mesostructured Silica Thin Films. J. Phys. Chem. C 2008, 112, 18895. [CrossRef]

18. Sun, B.; Wang, C.; Han, S.; Huc, Y.; Zhangd, L. Metal-enhanced fluorescence-based multilayer core-shell Ag-nanocube@SiO ${ }_{2} @ P M O s$ nanocomposite sensor for $\mathrm{Cu}^{2+}$ detection. RSC ADV 2016, 6, 61109. [CrossRef]

19. Xu, S.; Cao, Y.; Zhou, J.; Wang, X.; Wang, X.; Xu, W. Plasmonic enhancement of fluorescence on silver nanoparticle films. Nanotechnology 2011, 22, 275715. [CrossRef] [PubMed]

20. Xia, B.; He, F.; Li, L. Metal-enhanced fluorescence using aggregated silver nanoparticles. Colloids Surf. A 2014, 444, 9-14. [CrossRef]

21. Yang, B.; Lu, N.; Qi, D.; Ma, R.; Wu, Q.; Hao, J.; Liu, X.; Mu, Y.; Reboud, V.; Kehagias, N.; et al. Tuning the Intensity of Metal-Enhanced Fluorescence by Engineering Silver Nanoparticle Arrays. Small 2010, 6, 1038. [CrossRef] [PubMed]

22. Pang, J.; Theodorou, I.G.; Centeno, A.; Petrov, P.K.; Alford, N.M.; Ryan, M.P.; Xie, F. Gold nanodisc arrays as near infrared metal-enhanced fluorescence platforms with tuneable enhancement factors. J. Mater. Chem. C 2017, 5, 917-925. [CrossRef]

23. Theodorou, I.G.; Jawad, Z.A.R.; Q, J.; Aboagye, E.O.; Porter, A.E.; Ryan, M.P.; Xie, F. Gold Nanostar Substrates for Metal-Enhanced Fluorescence through the First and Second Near-Infrared Windows. Chem. Mater. 2017, 29, 6916-6926. [CrossRef]

24. Darvill, D.; Centeno, A.; Xie, F. Plasmonic fluorescence enhancement by metal nanostructures: Shaping the future of bionanotechnology. Phys. Chem. Chem. Phys. 2013, 15, 15709-15726. [CrossRef] [PubMed]

25. Deng, W.; Xie, F.; Baltar, H.T.M.C.M.; Goldys, E.M. Metal-enhanced fluorescence in the life sciences: Here, now and beyond. Phys. Chem. Chem. Phys. 2013, 15, 15695-15708. [CrossRef] [PubMed]

26. Xie, F.; Centeno, A.; Ryan, M.R.; Riley, D.J.; Alford, N.M. Au nanostructures by colloidal lithography: From quenching to extensive fluorescence enhancement. J. Mater. Chem. B 2013, 1, 536-543. [CrossRef]

27. Xie, F.; Pang, J.S.; Centeno, A.; Ryan, M.P.; Riley, D.J.; Alford, N.M. Nanoscale control of Ag nanostructures for plasmonic fluorescence enhancement of near-infrared dyes. Nano Res. 2013, 6, 496-510. [CrossRef]

28. Drozdowicz-Tomsia, K.; Xie, F.; Goldys, E.M. Deposition of Silver Dentritic Nanostructures on Silicon for Enhanced Fluorescence. J. Phys. Chem. C 2010, 114, 1562-1569. [CrossRef]

29. Prucnal, S.; Liu, F.; Voelskow, M.; Vines, L.; Rebohle, L.; Lang, D.; Berencén, Y.; Andic, S.; Boettger, R.; Helm, M.; et al. Ultra-doped n-type germanium thin films for sensing in the mid-infrared. Sci. Rep. 2016, 6, 27643. [CrossRef] [PubMed]

30. Stanley, R. Plasmonics in the mid-infrared. Nat. Photon 2012, 6, 409-411. [CrossRef]

31. Soref, R. Mid-infrared photonics in silicon and germanium. Nat. Photon 2010, 4, 495-497. [CrossRef]

32. Baldassarre, L.; Sakat, E.; Frigerio, J.; Samarelli, A.; Gallacher, K.; Calandrini, E.; Isella, G.; Paul, D.J.; Ortolani, M.; Biagioni, P. Midinfrared plasmon-enhanced spectroscopy with germanium antennas on silicon substrates. Nano. Lett. 2015, 15, 7225-7231. [CrossRef] [PubMed]

33. Frigerio, J.; Ballabio, A.; Isella, G.; Sakat, E.; Biagioni, P.; Bollani, M.; Napolitani, E.; Manganelli, E.; Virgilio, M.; Grupp, A.; et al. Tunability and losses of mid-infrared plasmonics in heavily doped germanium thin films. arXiv, 2016.

34. Bharadwaj, P.; Novotny, L. Spectral dependence of single molecule fluorescence enhancement. Opt. Express 2007, 15, 14266-14274. [CrossRef] [PubMed]

35. Centeno, A.; Xie, F.; Alford, N. Predicting the fluorescent enhancement rate by gold amd silver nanospheres using finite-difference time-domain analysis. IET Nanobiotechnol. 2013, 7, 50-58. [CrossRef] [PubMed]

36. Liaw, J.W.; Chen, C.S.; Chen, J.H. Plasmonic effect of gold nanospheres on spontaneous emission. Prog. Electromagn. Res. B 2011, 31, 283-296. [CrossRef] 
37. Aziz, U.A.; Rashid, N.N.; Aid, S.R.; Centeno, A.; Akira, S.; Ikenoue, H.; Xie, F. Electrical and Structural Analysis on the Formation of n-type Junction in Germanium. IOP Conf. Ser. Mater. Sci. Eng. 2017, 204, 012003. [CrossRef]

38. Aid, S.R.; Matsumoto, S.; Fuse, G. Boron diffusion behavior in silicon during shallow $\mathrm{p}+/ \mathrm{n}$ junction formation by non-melt excimer laser annealing. Phys. Status Solidi A 2011, 208, 1646-1651. [CrossRef]

39. Aid, S.R.; Matsumoto, S.; Fuse, G.; Sakuragi, S. Comparison of boron diffusion in silicon during shallow $\mathrm{p}+/ \mathrm{n}$ junction formation by non-melt excimer and green laser annealing. Phys. Status Solidi A 2011, 208, 2772-2777. [CrossRef]

40. Byrnes, S.J. Multilayer optical calculations. arXiv, 2016.

41. Centeno, A.; Ahmed, B.; Reehal, H.; Xie, F. Diffuse scattering from hemispherical nanoparticles at the air-silicon interface. Nanotechnology 2013, 24, 415402. [CrossRef] [PubMed]

42. Kuzmenko, A. Guide to RefFIT Software to Fit Optical Spectra. 2015. Available online: https:/ / sites.google. com/site/reffitprogram/home (accessed on 9 October 2017).

43. Oskooi, A.F.; Roundy, D.; Ibanescu, M.; Bernel, P.; Joannopoulos, J.D.; Johnson, S.G. MEEP: A flexible free-software package for electromagnetic simulations by the FDTD method. Comput. Phys. Commun. 2010, 181, 687-702. [CrossRef]

(C) 2018 by the authors. Licensee MDPI, Basel, Switzerland. This article is an open access article distributed under the terms and conditions of the Creative Commons Attribution (CC BY) license (http://creativecommons.org/licenses/by/4.0/). 